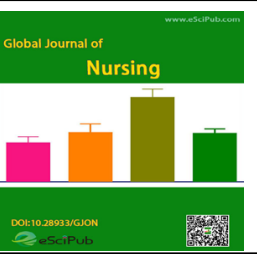

Global Journal of Nursing

(ISSN:2637-4994)

\title{
Safety Snacks: Improving Quality in Long-Term Care
}

\author{
${ }^{1}$ Leona Konieczny, DNP, MPH, RN-BC, CNE, ${ }^{2}$ Ashley Soyka, BSN, RN, LNHA \\ ${ }^{1}$ Central Connecticut State University, ${ }^{2}$ Bristol Health
}

\begin{abstract}
Falls among residents in the long-term care setting adversely affect resident health with potential for serious injury and quality of care provided. Nurses at the long-term care facility recognize the need to reduce falls. The nurses propose the intervention and name the initiative Safety Snacks. The research questions are: in *Correspondence to Author: a long-term care facility, does the practice of additional rounding ty, while providing snacks twice daily, decrease the incidence of falls and falls with serious injury as compared to not providing Safety Snacks over a three-month period? Will unintended weight loss change by additional rounding during which nourishment and fluids are given to residents twice daily over a three-month period? The study examines the number of falls, falls with serious injury, and unintended weight loss before and after the Safety Snacks Leona Konieczny, DNP, MPH, RNBC, CNE, 2Ashley Soyka, BSN, $\mathrm{RN}, \mathrm{LNHA}$

Central Connecticut State Universiintervention. Poissons Means Test and odds ratio are used for fall data analysis. A two-proportion z-test is used for weight loss data. The results demonstrate a reduction in falls and falls with serious injury. The odds ratio demonstrates a medium effect in falls with serious injury. The two-proportion test on unintended weight loss yields a significant difference of 0.09 with a $95 \%$ conHow to cite this article:

Leona Konieczny, Ashley Soyka. Safety Snacks: Improving Quality in Long-Term Care. Global Journal of Nursing , 2021, 4:24.

fidence interval of $(0.015,0.17)$. One implication is the increase in rounding while providing nutrition and hydration may reduce falls and falls with serious injury. The second implication is the more significant effect on unintended weight loss with providing Safety Snacks. The third implication is the empowerment of nurses to recommend and execute a change in practice to improve quality. Keywords: falls, long-term care, quality improvement, unintended weight loss
\end{abstract}


Falls in older adults may result in serious injury and poor outcomes. Consequences of falls may include fracture, acquired brain injury, fallophobia (post fall syndrome), decreased prefall mobility, and decreased baseline activity levels. Reduction in activity level may lead to loss of function and poor quality of life [1]. In addition to injury, falls among residents in longterm care may result in further deterioration from baseline, activity monitoring in the plan of care, and decreased rating of the facility. Falls in longterm care facilities are recognized as a safety and quality indicator. The Centers for Medicare and Medicaid (CMS) have core measures for quality and promote measurement that is evidence-based and generate information for quality improvement ${ }^{[2]}$. A strategy proposed by the nursing staff in long term care is the implementation of additional rounding twice daily with carts supplied with snacks and beverages. The residents would have extra observation and would be provided with nutrition and hydration outside regular meals.

\section{Literature Review}

\section{Nutrition and Hydration}

Osteoporosis and sarcopenia which are common in older adults have consequences resulting in frailty, falls, and fractures. The role of nutrition in preventing bone and muscle loss has been documented to improve function to reduce fall risk ${ }^{[1,3]}$. Nutrition and hydration was found to be one of the top three interventions in fall prevention ${ }^{[4]}$. Malnutrition risk is associated with a significant risk of a prospective fall ${ }^{[5]}$. Unmet nutritional needs can have a significant impact on musculoskeletal health and may have a direct influence on falls ${ }^{[6]}$.

\section{Cognitive Impairment}

Long term care residents are more likely to fall from bed and most falls occur in the resident room or the bathroom ${ }^{[7]}$. In one study, falls were frequent $(56.2 \%$ of the study sample) with dementia being highly predictive for fall ${ }^{[8]}$. A study with over 10,000 sample size identified cognitive impairment, physical frailty, and cognitive frailty for falls and fall-related fractures [9]. Persons with dementia fall more frequently and have more serious injury as compared to cognitively intact older adults ${ }^{[10] .}$

\section{Person-centered care}

While the literature contains information on alarm usage and fatigue in the critical setting, it was difficult to find research on the use of bed and chair alarms and alarm fatigue in long-term care. Alarms are reactive devices not proactive [11]. The literature supports a person centered approach to positively impact resident' rights, emotional, and physical well-being. A study done in long-term care using the PersonCentered Climate Questionnaire -Patient (PCQ$P)$ found person-centered care is an important feature for older adults and may prioritize this in respect to other elements [12]. Providing increased presence while providing hydration and/or nutrition at the bedside is person centered. The literature calls for innovative approaches with long term care residents to prevent falls ${ }^{[13]}$.

\section{Research Questions}

With residents in a long-term care facility, does the practice of additional rounding while providing snacks twice daily, decrease the incidence of falls and falls with serious injury as compared to not providing Safety Snacks over a three-month period? "Fall refers to unintentionally coming to rest on the ground, floor, or other lower level, but not as a result of an overwhelming external force (e.g., resident pushes another resident). An episode where a resident lost his/her balance and would have fallen, if not for staff intervention, is considered a fall" ${ }^{[14]}$. Serious injury is defined as a fracture or joint dislocation, laceration requiring suturing, and/or admission to acute care for treatment. The null hypothesis is there will be no difference in falls before and after the Safety Snacks intervention. Will unintended weight loss change by additional rounding during which nourishment and fluids are given to residents twice daily over a three-month period? Unintended weight loss is defined as $5 \%$ over 30 days or $10 \%$ over 180 
days. The null hypothesis is there will be no difference in unintended weight loss before and after the Safety Snacks intervention.

\section{Methodology}

The study site is a 128 bed long-term care facility. It is part of a small health system comprised of emergency services, outpatient medical offices, an acute care community hospital, a home care agency, and a long-term care facility. The site is located in a suburban city with a population of approximately 60,000 in the northeast United States of America. The facility has two nursing units on two floors totaling four units. Approximately $80 \%$ of residents at the study site have dementia. The facility is restraint free. Approval for the study is obtained from the Patient Care Services (PCS) Research Committee. Data includes the fiscal year 20202021. Fiscal year to date data is collected from 1 October 2020 through 30 June 2021. The number of falls and the number of falls per 1000 resident days is collected. Falls with serious injury and the number of falls per 1000 resident days is collected. Falls per 1000 resident days is consistent with benchmarking data from CMS. This parameter controls for any variation in census. All data is aggregate without any individual resident information. Two snack carts, one for each floor, are prepared twice daily by the Dietary Department. The snack carts are well stocked with a wide variety of foods and beverages. Resident diet orders were printed out by the nursing staff to confirm diet restrictions related to resident specific needs, ex. conditions such as dysphagia, diabetes, etc. The number of falls, falls per 1000 resident days, falls with serious injury, and falls with serious injury per 1000 resident days is collected from 1 July 2021 through 30 September 2021. Statistical analysis uses Poissons Means and odds ratio for fall data. A two proportion test is used for unintended weight loss data.

\section{Results}

The data shows there is decrease in number of falls, decrease in falls per 1000 resident days, decrease falls with serious injury, and decrease falls with serious injury per 1000 resident days. There are 56 falls during the study with a mean of 18.67 falls per month. This is a decrease from a 19.67 falls per month prior to the intervention. The falls per 1000 resident days are 5.39 post intervention as compared to 6.19 before the Safety Snacks. The falls with serious injury were 2 over three months with a mean of 0.67 as compared to 13 over nine months with a mean of 1.44 . The incidence of serious falls decreased by half. In examining, the falls with serious injury per 1000 resident days demonstrated approximately the same beneficial change. The mean per 1000 resident days for falls with serious injury was 0.19 with Safety Snacks as compared to a pre-intervention mean of 0.45 . See Safety Snacks Data, Table 1 for means of the data.

Table 1 Safety Snacks Means

\begin{tabular}{lll}
\hline & Pre & Post \\
\hline Falls per month & 19.67 & 18.67 \\
Falls per 1000 resident days & 6.19 & 5.39 \\
Falls with serious injury per month & 1.44 & 0.67 \\
Falls with serious injury per1000 resident days & 0.45 & 0.19 \\
\hline
\end{tabular}

The Poisson Mean Test at the $95 \%$ confidence interval shows the confidence intervals overlap (Pre: (16.9-22.8) and Post: (14.1-24.2)). The conclusion is that there is no significant difference in the number of falls per month pre to post. This does not change per 1000 days. Odds ratio is examined due to the small number of falls with serious injury. There is an overall medium 
effect on the odds ratio with the falls with serious injury (Odds Ratio: 0.25 ; s.e: 0.1268 ).

\section{Weight Loss Data}

Unintended weight loss is another indicator of quality in long-term care. Unintended weight loss is defined as $5 \%$ over 30 days or $10 \%$ over 180 days. The unintended weight loss from April through June 2021 was 15.1\%. With Safety Snacks, the unintended weight loss from July through September 2021 decreases to $5.88 \%$. A test of two proportions yielded a significant difference of 0.09 with a $95 \%$ confidence interval of $(0.015,0.17)$. This is discussed in the next section on implications on quality.

\section{Implications on Quality in Long-Term Care}

Residents have the right to expect and receive quality in long-term care. Reducing falls and more importantly reducing falls with serious injury increases the quality of life for the resident and the quality measured at the study site. While falls may be prevented, they are not totally avoidable in older adults requiring residential care. It is significant for falls with serious injury to be reduced in this study.

In addition to the fall prevention strategy, there was a statistically significant decrease in unintended weight loss. The carts were well stocked with a variety of cookies, muffins, granola bars, fruit cups, pudding, jello, ice cream. It was summer during the three months of the study. Fresh watermelon was provided and was a favorite snack for the residents. The cart was stocked with coffee, hot water for tea, water, and regular and sugar-free sodas. It was beneficial for the residents to have extra attention and to make a choice regarding their snacks. It is observed the residents enjoy additional interaction with staff. The increase in attention possibly increases the residents' quality of life. In some instances residents were having virtual visits with family. The virtual visits are related to visiting limitations due to COVID19 pandemic. The family or support persons' feedback is the snacks as favorable activity. The rationale for comparing three months pre- intervention rather than nine months is related to COVID-19. There were outbreaks in the fall and winter of 2020-2021 which may have been responsible for unintended weight loss. The decision is made to compare the months when no resident was actively ill from the virus.

The strengths of the study are an easily implemented intervention without a need for extra technology and the study may be replicated by other facilities. The limitations are the ability to generalize the findings beyond older adults in long-term care settings and the extra cost for nourishment. The other area to note is the possibility that not all intake was documented on the residents who were on intake and output monitoring.

There are further areas for study. One example is to compare nine months of data preintervention with nine months of fall data with Safety Snacks. Another area for further study, is specific findings related to unintended weight loss as this is the finding which showed the most change. Applicability to assisted living facilities might be an area for study by comparing resident attendance at "tea time" or "mocktail hour" and weight monitoring. Older People's Quality of Life (OPQoL)-brief scores may be studied in relation to improving care with Safety Snacks. A study in 2020 demonstrates the use of this instrument in long-term care. Their findings show 49.7\% report high Quality of Life (QoL). Modest QoL reported at $42.5 \%$ and $6.1 \%$ were reported as low [15]. There is improvement needed when half of the residents perceive quality of life as less than high.

Safety Snacks is an example of nursing innovation. The idea and name came from the nurses. The Executive Chef and the Dietary Department were consistent in providing the carts with food and drink. Many members of the facility participated in providing the snacks. This includes the Administrator, the Director of Nursing, the Resident Care Coordinator nurses, the Professional Development Specialist, Recreation Department personnel, and Certified Nursing Assistants (CNAs). Nurses at the study 
site are empowered to recommend and implement initiatives to provide quality improvement in long-term care.

\section{References}

[1]. Eliopoulos, C. Gerontological Nursing (10th ed.). Wolters Kluwer, 2022.

[2]. Quality measures. Centers for Medicare \& Medicaid Services. [Online] Available: https://www.cms.gov/Medicare/QualityInitiatives-Patient-AssessmentInstruments/NursingHomeQualitylnits/NHQIQu alityMeasures [Accessed Oct. 29, 2021].

[3]. Daly, R. M. Exercise and nutritional approaches to prevent frail bones, falls, and fractures: an update. Climacteric, vol. 20, pp. 119-124, 2017. https://doi.org/10.1080/13697137.2017.128689 0

[4]. Grealish, L., Real, B., Todd, J., Darch, J., Soltau, D., Phelan, M., Lunn, M., Brandis, S., Cooke, M., \& Chaboyer, W. Implementing evidence-based guidelines for falls prevention: observations of nursing activities during the care of older people with cognitive impairment. Worldviews on Evidenced-Based Nursing, vol. 16, pp. 335-343, 2019. https://doi.org/10.1111/wvn.12376

[5]. Eckert, C., Gell, N. M., Wingood, M., Schollmeyer, J., \& Tarleton, E. K. Malnutrition risk, rurality, and falls among communitydwelling older adults. Journal of Nutrition, Health, \& Ageing, vol. 25, pp. 624-627, 2021.

[6]. Walker, J. Nutrition and musculoskeletal health. British Journal of Community Nursing, vol. 25 (Sup8), pp. S30-S32, 2020. https://doi.org/10.12968/bjcn.2020.25.Sup8.S3 0

[7]. McArthur, C., Gonzalez, D. A., Roy, E., \& Giangregorio, L. What Are the circumstances of falls and fractures in long-term care? Canadian Journal on Aging, vol. 35, pp. 491-498, 2016. https://doi.org/10.1017/S0714980816000556

[8]. Cameron, E. J., Bowles, S. K., Marshall, E. G., \& Andrew, M. K. Falls and long-term care: a report from the care by design observational cohort study. BMC Family Practice, vol. 19, no. 73, 2018. https://doi.org/10.1186/s12875-0180741-6

[9]. Tsutsumimoto, K., Doi, T., Makizako, H., Hotta, R., Nakakubo, S., Makino, K., Suzuki, T., \& Shamada, H. Cognitive frailty is associated with fall-elated fracture among older people. Journal of Nutrition, Health \& Aging, vol. 22, pp. 1216-
1220, 2018. https://doi.org/10.1007/s12603018-1131-4

[10]. Fernando, E., Fraser, M., Hendriksen, J., Kim, C., \& Muir-Hunter, S. W. Risk factors associated with falls in older adults with dementia: a systematic review. Physiotherapy Canada, vol. 69, pp. 161-173, 2017. https://doi.org/10.3138/ptc.2016-14

[11]. Replacing alarms with person-centered approaches. CNA Training Advisor, vol. 16, pp. 2-4, 2018.

[12]. Kelly, F., Reidy, M., Denieffe, S., \& Madden, C. Older adults' views on their person-centered care needs in a long-term care setting in Ireland. British Journal of Nursing, vol. 28, pp. 552-557, 2019.

https://doi.org/10.12968/bjon.2019.28.9.552

[13]. Chan, D. K., \& Chan, L. K. Falls in nursing homes: challenges from a nursing perspective. British Journal of Community Nursing, vol. 24, pp. 6-9, 2019. https://doi.org/10.12968/bjcn.2019.24.1.6

[14]. CMS Measures Inventory Tool. Centers for Medicare \& Medicaid Services, 2021. [Online] Available:

https://cmit.cms.gov/CMIT_public/ViewMeasure ?Measureld=5285 [Accessed Oct. 29, 2021].

[15]. Haugan, G., Drageset, J., Andre, B., Kukulu, K., Mugisha, J., \& Utvaer, B. K. Assessing quality of life in older adults: psychometric properties of the OPQoL-brief questionnaire in a nursing home population. Health and Quality of Life Outcomes, vol. 18, no. 1, 2020. https://doi.org/10.1186/s12955-019-1245-3 